\title{
The 10 P's of Stroke: a Simple Strategy for Understanding Stroke Etiology and Management
}

\author{
Laurie Schluter MSN, FNP, ANVP ${ }^{1}$, Joe Tarsia MD ${ }^{1}$, Alyana Samai BHS ${ }^{1,2}$ \\ Sheryl Martin Schild, MD, PhD $^{1}$ \\ ${ }^{1}$ Stroke Program, Department of Neurology, Tulane University School of Medicine, New Orleans, 70112 \\ ${ }^{2}$ Department of Epidemiology, Tulane School of Public Health and Tropical Medicine, New Orleans, 70112
}

\begin{abstract}
The evaluation and management of strokevaries between patients due to differences in individual physiology and disease progression. The goal of this article is to provide health care professionals with a comprehensive resource that adequately catalogs the causes and physiological mechanisms of stroke, as well as diagnostic testing techniques and effective therapeutic interventions relevant to stroke care. This article examines what we consider at our center to be the core factors in ischemic stroke that areconsidered for each patient to deliver effective individualized care. These variables form a vital patient profile summarized in a mnemonic called the "P's of Stroke". The "P's" encompass the influence and significance of cardiac dysfunction (pump), blood pressure, perfusate, arterial blood flow/vasculature (pipes), cerebralblood flow (plumbing), perfusion, parenchyma, penumbra, physical rehabilitation and recovery, and prevention with relation to acute ischemic stroke (AIS) assessment and treatment
\end{abstract}

Keywords: Stroke, Ischemic, Thrombi, Clot, Evaluation, Management, Brain, Blood Flow

\section{INTRODUCTION}

The aim of this article is to provide healthcare providers with a manageable resource that helps explain the etiology, pathophysiology, and various diagnostic testing and therapeutic interventions that take place while caring for a stroke patient. The 10 P's of stroke link the core pathophysiology of an acute ischemic stroke (AIS) with the practical management of the patient, aiding in the understanding and communication of why the management of one patient may not be the same as the next.

The P's of stroke were first introduced in 2001 by Howard A. Rowley MD, who described the four concepts of parenchyma, pipes, perfusion and penumbra in regards to stroke imaging(Rowley, 2001). The addition of a fifth $\mathrm{P}$ prevention of complications - was described by Felberg and Naidech in looking at treatment for AIS(Felberg \& Naidech, 2003). There are several sub-classifications of ischemic stroke, including intracerebral hemorrhage and subarachnoid hemorrhage, which are beyond the scope of this article. Still, it is important to realize that once a stroke has occurred, it is vitally important to determine the underlying cause so that it can be adequately treated. This article should provide healthcare providers with an effective tool to help educate their patients and family members during the process of undergoing numerous, and sometimes rigorous, diagnostic tests and interventions.

$\mathbf{1}=$ "PUMP"- The heart acts as the "pump" for blood distribution throughout the body, and aids the delivery of oxygen and glucose to the brain for adequate energy metabolism and function. Testing methodologies for the evaluation of the "pump" include echocardiography, either transthoracic or transesophageal, and 12-lead electrocardiogram.

An important component of a thorough cardiac workup is examining cardiac output, which is integrally related to stroke volume and heart rate. Deficiencies in either of these components may contribute to an overall decrease in cerebral perfusion pressure resulting in global hypoxemic-ischemic brain injury or focal cerebral ischemia. Focal ischemia may occur when perfusion pressure generated by the heart falls below a critical threshold in order to circumvent a critical stenosis in a large vessel (i.e.: carotid artery stenosis)and may lead to characteristic watershed infarcts.

Also essential to the cardiac workup is detection for the presence of thrombi than can serve as a source of emboli. Cardioembolic stroke, caused by the dislodging of a clot formed in the heart, makes up approximately 14-30\%of all ischemic strokes(Arboix \& Alió, 2010). While, most cardiac thrombi originate within the left atrium or

\footnotetext{
${ }^{1}$ Corresponding Author: asamai@tulane.edu
} 
ventricle(Sharma, McCullough, Philbin, \& Weaver, 2000), several defects in cardiac structure or function, including atrial fibrillation or reduced left ventricular ejection fraction, increase the risk for formation of thrombi. Emboli can also originate on the valvular structuresand can be eitherinfectious (septic emboli) or non-infectious in nature.

A patent foramen ovale (PFO) with or without an associated atrial septal aneurysm (ASA) illustrates the concept of paradoxical emboli as a cardioembolic stroke risk factor. If a PFO or other source for right(venous) to left (arterial) shunting is found within the heart, a workupto determine the source of deep vein thrombosis(DVT) should be completed in addition to a venous hypercoagulable work-up, depending on other patient characteristics. In some cases, a PFO or ASA could be considered to be the thrombogenic source.

The most common diagnostic tests to evaluate the heart areelectrocardiography (ECG) and echocardiography. Traditionally, echocardiograms are performed with a transthoracic approach (TTE), which allows for visualization of the ejection fraction and overall heart function, the presence of chronic hypertensive heart disease as evidenced by diastolic dysfunction or left ventricular hypertrophy, and valvular dysfunction or malformation. A "bubble study" may be also be performed as a component of the echocardiogram by injecting agitated saline or contrast through the venous circulation to detect visualization of the saline bubbles passing through to the left side of the heart in the presence of an intracardiac shunt. Evidence of other entities mentioned above such as thrombi or valvular vegetations may be visible if large enough, but these entities are usually clarified or further characterized by transesophageal (TEE) viewing. TEE allows for a higher resolution and is better to detect not only thrombi and evidence of blood stagnation, but also entities such as an atrial septal defect (ASD) or PFO and is often necessary to detect clots in the posterior projecting left atrial appendage or atheroma in the aortic arch(Black, Hopkins, Lee, Jacobson, \& Walsh, 1991)

$\mathbf{2}=$ "PRESSURE" - Blood pressure is the driving force for delivering oxygen to the brain and is a key component of maintaining adequate cerebral perfusion pressure, determined by the difference in the mean arterial pressure (MAP) and intracranial pressure (ICP). Decrements in MAP or increases in ICP may compromise cerebral perfusion and lead to brain ischemia.

Elevated blood pressure at the time of AIS is common. The 2013 American Heart Association/American Stroke Association (AHA/ASA) guidelines recommend permissive hypertension during the acute phase of an ischemic stroke with the theoretical benefit of maintaining cerebral perfusion pressure to tissue that may depend on delayed flow through collaterals. The numbers in this guideline suggest a goal of SBP $<220 \mathrm{mmHg}$ an DBP $<120 \mathrm{mmHg}$ during this time period. If intraventricular tissue plasminogen activator(IV tPA) is administered, the goal is $<180 / 105$ $\mathrm{mmHg}$ during the 24 hours post-administration. The lower hemorrhagic complications with tighter control of blood pressure was seen in the earlier trials of thrombolysis for acute myocardial infarction and in retrospective analyses of post-tPA brain hemorrhages after treatment for ischemic stroke (Lyerly et al., 2014).If necessary, infusions with nicardipine or Labetalol may be used to maintain these parameters(Adams et al., 1993).

To date, there is no substantial evidence regarding when to begin to control blood pressure in the immediate poststroke phase and when to initiate or restart long-term anti-hypertensives. Furthermore, limited data is available to suggest specific target ranges of blood pressure control or specific agents to prevent further strokes. Long-term blood pressure target levels remains unclear, but guidelines provided by the Joint National Committee on Prevention, Detection, Evaluation, and Treatment of High Blood Pressure should be followed regarding a normal blood pressure of 120/80 $\mathrm{mmHg}$ (Chobanian et al., 2003). Additionally, the optimal drug regimen remains uncertain, but available data supports the use of angiotensin converting enzyme inhibitors (ACE-I) or angiotensin receptor blockers (ARB) as first-line treatment if no contraindications are present(Sacco et al., 2006).

3 ="PERFUSATE" -Perfusate is the liquid that moves through a solid organ. With respect to stroke, perfusate is the blood.

Two criteria must be satisfied for the perfusate to function normally in the body: 1) the blood must carry sufficient oxygen and glucose, without being too viscous or prone to clotting and 2) sufficient blood volume must be present to mount the necessary pressure for adequate blood flow throughout the body.

Oxygen is necessary in key metabolic cellular functions to maintain viability; therefore, hypoxia can cause ischemiaon a local level with a vascular occlusion and on a global level with a reduction in CPP. Additionally, the amount of glucose in the blood is crucial in the setting of stroke. Insufficient glucose levels can augment acidosis in the ischemic tissue, a process that is toxic to the already compromised brain cells(Auer, 2004). Conversely, elevated glucose is associated with increased infarct volume, risk of hemorrhagic conversion, and poor outcome(Gentile, Seftchick, Huynh, Kruus, \& Gaughan, 2006). Whether hyperglycemia is a modifiable risk factor for these adverse 
events or a marker for poor outcome has not yet been determined. The recently completedin the Stroke Hyperglycemia Insulin Network Effort (SHINE) trial may lead to better understanding of best practice once the results are released(Southerland \& Johnston, 2012). Euglycemia is the current goal in acute stroke management.

Increased viscosity of blood occurs as a result ofsickle cell anemia, polycythemia vera, essential thrombocytosis, acute myelogenous leukemia, and during various treatments such as immune globulin administration. This increase in viscosity canincrease the risk for ischemic strokes due to sludging in the microcirculation.Some patients, particularly those who are young, with a paucity of traditional vascular risk factors, may have gene mutations or abnormal levels ofvarious clotting factors. Hypercoagulable workupsshould be considered in the case of stroke in young patients (<50years old), those with recurrent cryptogenic stroke, or associated diseases (autoimmune, cancer, etc.), Laboratory analysis for known arterial or venous clotting abnormalities, such as in the presence of an intracardiac shunt or when venous sinus thrombosis is the etiology of ischemic stroke,may be warranted in these patients.

$4=$ "PIPES" - The arteries that carry blood to the brain.

The goal in "pipe" management is to prevent endothelial dysfunctioncaused by hypertension, smoking, diabetes, and hyperlipidemia.Endothelial dysfunction can lead to activation of coagulation causing platelet adherence, aggregation, and clot formation(Levi, van der Poll, \& Büller, 2004).Atherosclerosis and atherothrombosis can result from damage to the arteries ultimately causing a blockage. When stenosis is identified in extracranial vessels, stenting and surgery are considerations for prevention of stroke.

Ulcerations, dissections, inflammation (vasculitis), vasospasm, and Moyamoya disease are all arterial abnormalities. Vascular imaging such as CTangiography (CTA), MR angiography(MRA), or catheter angiography is often used to diagnose these conditions. Determining what testing is appropriate relies on individual patient risk factors, institutional availability, and whether or not the need for intervention is anticipated. Carotid ultrasound is noninvasive andless expensive than some other forms of testing;however itis technician and anatomy dependent, and contributes almost no information in theexamination of the posterior circulation. CTA, which evaluates the vessel lumenusing contrast as a surrogate marker for blood,can be quickly obtained. CTArequires a large-bore intravenous catheter, harbors the risk of contrast-related allergy or kidney dysfunction in relation to glomerular filtration rate (GFR), and has reduced signal-to-noise at bony interfaces and in the presence of dense calcium deposits in the arterial wall. MRA is dependent on flow and is of limited value if the patient moves. It requires contrast for extracranial vessels, but not for intracranial, so the intracranial vessels may still be imaged (supplemented by ultrasound for the carotid arteries) when kidney function or IV access is an issue. Catheter angiography provides the highest resolution vessel imaging and allows for determination of collateral blood supply and permits intervention, but it is also the most invasive and expensive of the vessel imaging techniques.

Vasospasm may be suspected in patients with sympathomimetic exposure, such as cocaine (Esse, Fossati- Bellani, Traylor, \& Martin- Schild, 2011), or recent subarachnoid hemorrhage. Transcranial Doppler (TCD) is a techniciandependent,noninvasive test to monitor velocities and patterns of blood flow in the intracranial pipes and is most commonlyused for screening for vasospasm. Catheter angiography may be used to confirm and treat vasospasm, which can include injection of calcium channel blockers or angioplasty(Macdonald, 2006). TCD can detect an acute occlusive intracranial thrombus and continuous TCD monitoring has been shown to enhance thrombolysis (Alexandrov, Wojner, \& Grotta, 2004). TCD can also be used to detect risk of stroke in patients with sickle cell disease and the potential need for exchange transfusions to reduce this risk(Lee et al., 2006).

$5=$ "PLUMBING"- describes the communication of arteries in the brain; it is how the pipes are connected.

Not everyone has the same configuration of arteries; however, if there is good "plumbing," the damage from loss of a single vesselmay be minimized. Good plumbing provides a natural bypass when one pipe becomes obstructed. In this situation, occlusion of an artery,even a carotid artery, can be clinically silent and without brain injury. Fetal vessels, communicating vessels between anterior and posterior circulation,may be present and collateral circulation may have developed, which can allow for decreased risk or extent of infarct.

Poor plumbing makes areas of the brain dependent on a single artery for blood flow to a discrete region. In this situation, occlusion of an artery can cause total territorial infarction and can be devastating. In some situations, such as Moyamoya disease, improved plumbing is created through surgical intervention by connecting an artery from outside of the skull to one inside of the skull to provide a bypass and supply blood to the brain(Mesiwala, Sviri, Fatemi, Britz, \& Newell, 2008). 
6 = "PERFUSION" - the movement of a liquid (perfusate/blood) through a tissue (parenchyma/brain).

Multiple factors impact perfusion - cardiac output, blood pressure, blood viscosity, pipe narrowing or occlusion (all described above), and venous return. Head-of-bed positioning can play a part with flattened head-of-bed promoting perfusion to the brain(Wojner-Alexander, Garami, Chernyshev, \& Alexandrov, 2005). Raising the head-of-bed can decrease perfusion and intracranial pressure (ICP) in the setting of elevated ICP and hemorrhagic strokes.

Venous outflow is also another component of this P. Placement of internal jugular catheters should be avoided or compromise of proper venous drainage may result.

Perfusion imaging through CT with contrast can target a suspected ischemic area in the brain and can measure the cerebral blood volume, cerebral blood flow, and time it takes for contrast to be delivered, distributed, and drained to and from the brain. Comparison to a non-ischemic area may be useful in determining if there may still be salvageable brain tissue during ischemia. This is particularly relevant when considering thrombolytic treatment in patients outside of the FDA or AHA/ASA recommendedtime frame for consideration of intra-arterial therapies, like clot removal. MRI with perfusion allows for wholebrain imaging, while most CT scanners and perfusion software forces the treating physician to guess where the key information can be obtained in a limited number of brain slices. MRI with perfusion imaging ismore time consuming and cannot be performed in patients with pacemakers, defibrillators, and reduced glomerular filtration rates due to the risk of nephrogenic systemic sclerosis. The ultimate perfusion imaging would be achieved with a catheter angiogram, which detects the distribution of contrast within the parenchyma as evidence of perfusion.

7 = "PARENCHYMA" -is the brain tissue.

Computed tomography (CT) and magnetic resonance imaging (MRI) are the most frequent types of parenchymal imaging. CT imaging can show hyperacute signs of stroke suchas hyperdense arteries, sulcal effacement, and loss of differentiation between gray and white matter, but it can take as long as 4.5 hours to demonstrate a new stroke. CT imaging of the head is highly sensitive for acute intracranial hemorrhage(Wong, Siu, Abrigo, Ahuja, \& Poon, 2012). Evaluation of the brain with MRI, however, is more sensitive and specific than CT in evaluating parenchyma(Kidwell et al., 2004).CT angiography can detect vascular anomalies and active bleeding. Post-contrast brain CT images can identify underlying hemorrhagic lesions and slower oozing of blood within the hematoma, as contrast is identified as a higher density material than blood. MRI is also useful in identifying the age of hemorrhage based on combinations of signal intensity on T1 and T2 sequences(Park et al., 2010). MRI is risky when performed in the first few hours of ICH when active bleeding and clinical worsening is most likely, particularly since the patient must lie flat and monitoring of mentation is compromised.

Imaging sequences include 1) diffusion weighted imaging (DWI), which can show stroke within minutes of ischemia, 2) fluid-attenuated inversion recovery (FLAIR) imaging, which can help identify burden of disease by showing old infarcts and white matter disease, buttakes about 4.5 hours to demonstrate a new area of ischemic tissue and 3) gradient Echo (GRE) imaging, which can identify areas of blood product deposition to help detect hemorrhage and microbleeds.

$8=$ "PENUMBRA" -is the brain tissue that is ischemic, but not yet dead.

Within the penumbra, blood flow is low enough that the tissue cannot serve its particular function, but is still capable of performing cellular survival steps. The penumbra is the target when recanalization strategies or neuroprotection is attempted. The ratio of penumbra to infarct declines with each passing minute during a stroke which is why treatment with recanalization strategies is so time-sensitive. The major determinants of whether a brain cell dies include the depth of the ischemia and the duration of ischemia. If blood flow is severely reduced, brain cells die rapidly, penumbra will be lost, and the stroke will complete much faster than if is there is only a mild reduction in flow. Once the tissue is infarcted, the cells cannot even complete self-survival steps and kill themselves in a process called apoptosis. If recanalization occurs in a completed infarct, the patient is exposed to risk of brain bleeding due to reperfusion injury withoutany benefit of restoring blood flow to dead brain.

The presence or absence of penumbra can be determined by perfusion imaging. The volume of penumbra can be determined by the proportion of brain experiencing a reduction in blood flow without severe reduction in blood volume. In the penumbra, the microcirculation and collaterals are dilated, resulting in an increase in blood volume, despite a reduction in blood flow. Identifying the presence of a penumbra may contribute to decisionmaking for extended window thrombolysis or endovascular intervention, but the penumbra may be lost if recanalization doesn't occur rapidly. 
9 = "PHYSICAL REHABILTATION \& RECOVERY" -is crucialimmediately following stabilization of stroke being that stroke is the leading cause of adult disability.

Following a stroke, the brain is capable of remodeling and can be impacted by therapeutic interventions. This includes physical therapy (PT), occupational therapy (OT), and speech/ language pathology (SLP).Therapies should be initiated during the acute hospitalization once the patient is able to actively participate.

Early mobilization was studied in A Very Early Rehabilitation Trial for stroke(AVERT) and was found to be safe at 24 hours post-acute stroke(Bernhardt, Dewey, Thrift, Collier, \& Donnan, 2008). Increasing activity can decrease risk of complications such as venous thromboembolism, pneumonias, and skin breakdown and can promote a more rapid recovery. A multidisciplinary approach should be used to determinethe appropriate setting for the patient's next level of care based on their individual medical, rehabilitation, and personal needs at the time of discharge. Options include outpatient therapies in outpatient centers or through home health services, skilled nursing facilities and nursing homes, or in an inpatient setting including long-term acute care (LTAC) hospitals or inpatient rehabilitation facilities (IRF). Assistive devices for mobility may assist in achieving increased independence. Customized splints may be recommended for positioning of limbs affected by paresis or paralysis.

$10=$ "PREVENTION" -Prevention of stroke could significantly reduce deaths and disability.

Awareness of risk factors through routine medical checkups is needed for effective primary prevention of cerebrovascular disease. Once these risk factors are identified, methods to reduce risk should be recommended by healthcare providers and carried out by the individual at risk. This may include management of chronic diseases with vascular implications such as hypertension, diabetes, and dyslipidemias. Hypertension is the number one risk factor for stroke(Staessen, Kuznetsova, \& Stolarz, 2003)and lowering blood pressure to values below 120/80 mmHg can reduce this risk(Chobanian et al., 2003). This can be achieved with lifestyle changes, such as reducing dietary intake of sodium and losing weight, along with possible addition of medications. Diabetes leads to significant microvascular and macrovascular effects and requires self-care, including blood sugar monitoring and control of blood glucose to euglycemic range with the use of dietary and pharmaceutical interventions. Dyslipidemias including elevations in triglycerides and/or low density lipoproteins (LDL) or lowhigh density lipoproteins (HDL) can have negative effects on the arteries and can be managed with lifestyle modifications including dietary changes, weight loss, and exercise along with medications if needed. Awareness of family history of these vascular risk factors should be taken seriously and healthy lifestyle with monitoring of weight, dietary intake and activity should be instituted along with laboratory monitoring.

At age 50, antiplatelet medications should be considered for reduction of vascular events due to abnormal platelet aggregation with advancing age(Berger et al., 2006). Patients with known history of clotting disorders, unprovoked DVT or pulmonary embolism (PE), mechanical heart valves, cardiac ejection fraction $<30 \%$, or atrial fibrillation may be considered for anticoagulant therapy for reduction of embolic stroke risk.Patients with constant or paroxysmal atrial fibrillation experienced increased stroke risk if they are over age 75, or have hypertension, diabetes, congestive heart failure or previous stroke(Odum, Cochran, Aistrope, \& Snella, 2012). If an individual exhibits signs of obstructive sleep apnea, a sleep study should be performed and therapeutic intervention should be prescribed if the disorder is identified.

Healthy living including maintaining a BMI below 20.0-24.9 (per the NIH), keeping physically active with regular exercise, reducing emotional stress and achieving restful sleep can promote well-being. Avoidance of smoking, illicit drug use and alcohol consumption in excessive amounts can reduce incidence of stroke.

Once an individual has experienced a transient ischemic attack or a completed stroke, secondary prevention should be instituted with use of an antithrombotic daily and goals of blood pressure $<120 / 80 \mathrm{mmHg}$, triglycerides $<150$ $\mathrm{mg} / \mathrm{dL}, \mathrm{HDL}>45 \mathrm{mg} / \mathrm{dL}, \mathrm{LDL}<70 \mathrm{mg} / \mathrm{dL}$, and blood glucose in normal range. Depending on the etiology found for the patient's stroke, they may need other individualized treatments including carotid intervention, PFO closure, CHF management, or exchange transfusions in the case of sickle cell anemia or polycythemia vera(Grima, 2000).

\section{SUMmARY}

Evaluation, treatment, prevention and management of a stroke can be quite complicated. Finding a method of understanding the multiple facets of stroke can be challenging when trying to cover a broad audience including medical students, doctors, nurses, allied health personnel and lay persons. Using the $10 \mathrm{P}$ 's of stroke as a basis for this education can lead to a systematic way of putting together all of the factors which come into play. Healthcare providers have both a responsibility and an opportunity to provide patient education in a way that can be brought to the level of understanding for that particular patient and their loved ones. This can have a major impact on their 
ability to follow their recommended treatment plan. Providing a method of learningabout stroke that is easier to understand may give healthcare providers the tools to enhance their ability to optimally care for these patients and to provideeffective patient education.

\section{The 10 P's of Stroke -}

Causes, Pathophysiology, Evaluation, and Management Tool

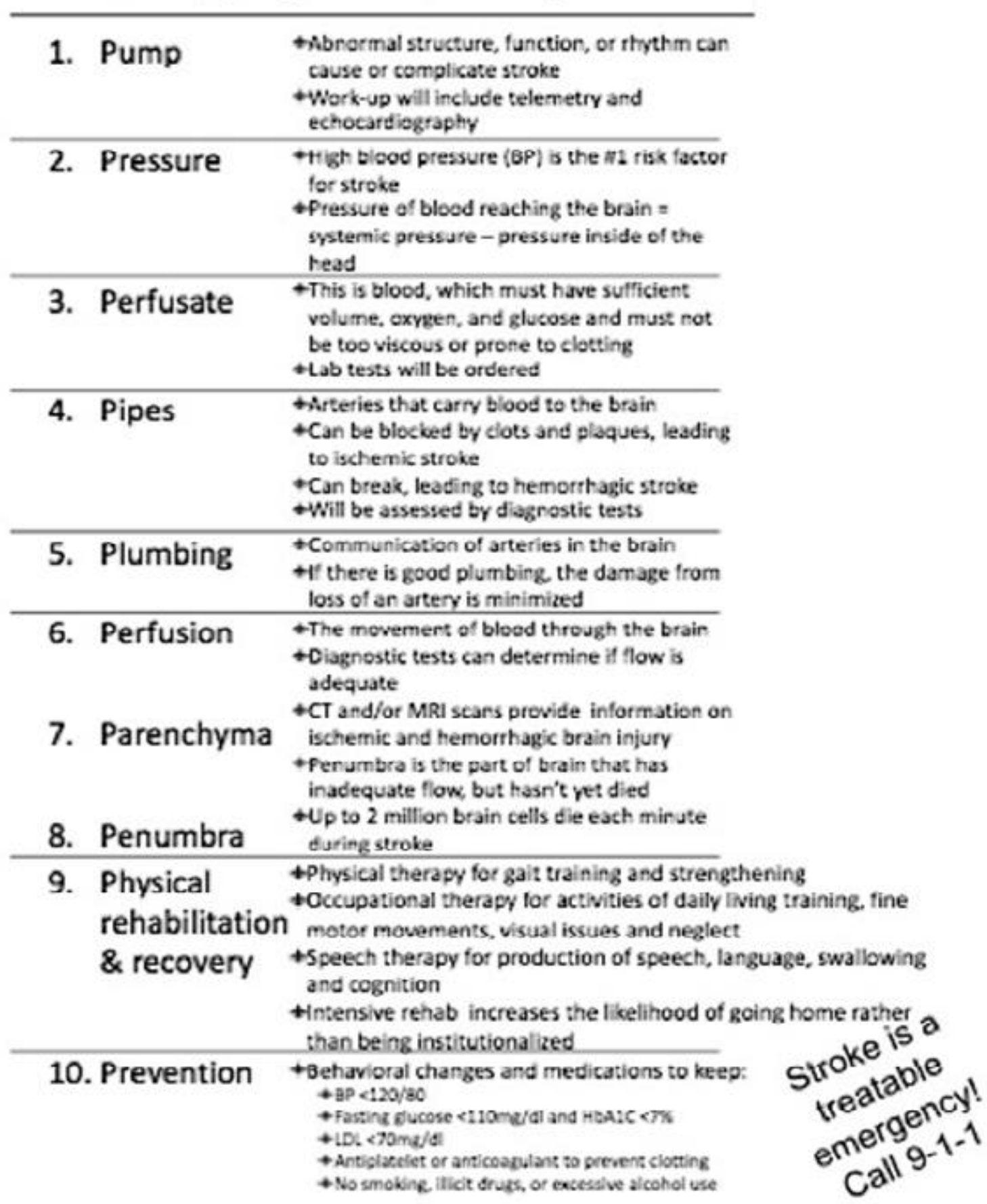

Fig1. The 10 P's of Stroke Reference Guide 


\section{REFERENCES}

[1] Adams, H. P., Bendixen, B. H., Kappelle, L. J., Biller, J., Love, B. B., Gordon, D. L., \& Marsh, E. E. r. (1993). Classification of subtype of acute ischemic stroke. Definitions for use in a multicenter clinical trial. TOAST. Trial of Org 10172 in Acute Stroke Treatment. Stroke, 24(1), 35-41.

[2] Alexandrov, A. V., Wojner, A. W., \& Grotta, J. C. (2004). CLOTBUST: Design of a Randomized Trial of Ultrasound- Enhanced Thrombolysis for Acute Ischemic Stroke. Journal of Neuroimaging, 14(2), 108-112.

[3] Arboix, A., \& Alió, J. (2010). Cardioembolic stroke: clinical features, specific cardiac disorders and prognosis. Current cardiology reviews, 6(3), 150.

[4] Auer, R. N. (2004). Hypoglycemic brain damage. Forensic science international, 146(2), 105-110.

[5] Berger, J. S., Roncaglioni, M. C., Avanzini, F., Pangrazzi, I., Tognoni, G., \& Brown, D. L. (2006). Aspirin for the primary prevention of cardiovascular events in women and men: a sex-specific meta-analysis of randomized controlled trials. Jama, 295(3), 306-313.

[6] Bernhardt, J., Dewey, H., Thrift, A., Collier, J., \& Donnan, G. (2008). A very early rehabilitation trial for stroke (AVERT) phase II safety and feasibility. Stroke, 39(2), 390-396.

[7] Black, I. W., Hopkins, A. P., Lee, L. C., Jacobson, B. M., \& Walsh, W. F. (1991). Role of transoesophageal echocardiography in evaluation of cardiogenic embolism. British heart journal, 66(4), 302-307.

[8] Chobanian, A. V., Bakris, G. L., Black, H. R., Cushman, W. C., Green, L. A., Izzo, J. L., Jones, D. W., Materson, B. J., Oparil, S., \& Wright, J. T. (2003). Seventh report of the joint national committee on prevention, detection, evaluation, and treatment of high blood pressure. Hypertension, 42(6), 1206-1252.

[9] Esse, K., Fossati- Bellani, M., Traylor, A., \& Martin- Schild, S. (2011). Epidemic of illicit drug use, mechanisms of action/addiction and stroke as a health hazard. Brain and behavior, 1(1), 44-54.

[10] Felberg, R. A., \& Naidech, A. M. (2003). The 5 Ps of acute ischemic stroke treatment: Parenchyma, pipes, perfusion, penumbra, and prevention of complications. Southern medical journal, 96(4), 336-342.

[11] Gentile, N. T., Seftchick, M. W., Huynh, T., Kruus, L. K., \& Gaughan, J. (2006). Decreased mortality by normalizing blood glucose after acute ischemic stroke. Academic emergency medicine, 13(2), 174-180.

[12] Grima, K. M. (2000). Therapeutic apheresis in hematological and oncological diseases. Journal of clinical apheresis, 15(1- 2), 28-52.

[13] Kidwell, C. S., Chalela, J. A., Saver, J. L., Starkman, S., Hill, M. D., Demchuk, A. M., Butman, J. A., Patronas, N., Alger, J. R., \& Latour, L. L. (2004). Comparison of MRI and CT for detection of acute intracerebral hemorrhage. Jama, 292(15), 1823-1830.

[14] Lee, M. T., Piomelli, S., Granger, S., Miller, S. T., Harkness, S., Brambilla, D. J., \& Adams, R. J. (2006). Stroke Prevention Trial in Sickle Cell Anemia (STOP): extended follow-up and final results. Blood, 108(3), 847-852.

[15] Levi, M., van der Poll, T., \& Büller, H. R. (2004). Bidirectional relation between inflammation and coagulation. Circulation, 109(22), 2698-2704.

[16] Lyerly, M. J., Albright, K. C., Boehme, A. K., Bavarsad Shahripour, R., Houston, J. T., Rawal, P. V., Kapoor, N., Alvi, M., Sisson, A., \& Alexandrov, A. W. (2014). Safety of protocol violations in acute stroke tPA administration. Journal of Stroke and Cerebrovascular Diseases, 23(5), 855-860.

[17] Macdonald, R. L. (2006). Management of cerebral vasospasm. Neurosurgical review, 29(3), 179-193.

[18] Mesiwala, A. H., Sviri, G., Fatemi, N., Britz, G. W., \& Newell, D. W. (2008). Long-term outcome of superficial temporal artery-middle cerebral artery bypass for patients with moyamoya disease in the US.

[19] Odum, L. E., Cochran, K. A., Aistrope, D. S., \& Snella, K. A. (2012). The CHADS2 versus the New CHA2DS2- VASc Scoring Systems for Guiding Antithrombotic Treatment of Patients with Atrial Fibrillation: Review of the Literature and Recommendations for Use. Pharmacotherapy: The Journal of Human Pharmacology and Drug Therapy, 32(3), 285-296.

[20] Park, S. Y., Kong, M. H., Kim, J. H., Kang, D. S., Song, K. Y., \& Huh, S. K. (2010). Role of'spot sign'on CT angiography to predict hematoma expansion in spontaneous intracerebral hemorrhage. Journal of Korean Neurosurgical Society, 48(5), 399-405.

[21] Rowley, H. A. (2001). The four Ps of acute stroke imaging: parenchyma, pipes, perfusion, and penumbra. American Journal of Neuroradiology, 22(4), 599-601. 
[22] Sacco, R. L., Adams, R., Albers, G., Alberts, M. J., Benavente, O., Furie, K., Goldstein, L. B., Gorelick, P., Halperin, J., \& Harbaugh, R. (2006). Guidelines for Prevention of Stroke in Patients With Ischemic Stroke or Transient Ischemic Attack A Statement for Healthcare Professionals From the American Heart Association/American Stroke Association Council on Stroke: Co-Sponsored by the Council on Cardiovascular Radiology and Intervention: The American Academy of Neurology affirms the value of this guideline. Circulation, 113(10), e409-e449.

[23] Sharma, N. D., McCullough, P. A., Philbin, E. F., \& Weaver, W. D. (2000). Left ventricular thrombus and subsequent thromboembolism in patients with severe systolic dysfunction. CHEST Journal, 117(2), 314-320.

[24] Southerland, A. M., \& Johnston, K. C. (2012). Considering hyperglycemia and thrombolysis in the Stroke Hyperglycemia Insulin Network Effort (SHINE) trial. Annals of the New York Academy of Sciences, 1268(1), 72-78.

[25] Staessen, J. A., Kuznetsova, T., \& Stolarz, K. (2003). Hypertension prevalence and stroke mortality across populations. Jama, 289(18), 2420-2422.

[26] Wojner-Alexander, A. W., Garami, Z., Chernyshev, O. Y., \& Alexandrov, A. V. (2005). Heads down Flat positioning improves blood flow velocity in acute ischemic stroke. Neurology, 64(8), 1354-1357.

[27] Wong, G. K. C., Siu, D. Y. W., Abrigo, J. M., Ahuja, A. T., \& Poon, W. S. (2012). Computed tomographic angiography for patients with acute spontaneous intracerebral hemorrhage. Journal of Clinical Neuroscience, 19(4), 498-500. 